\title{
Panic Disorder with Agoraphobia
}

National Cancer Institute

\section{Source}

National Cancer Institute. Panic Disorder with Agoraphobia. NCI Thesaurus. Code C97194.

A disorder in which an individual experiences recurrent, unexpected panic attacks and persistent concern about having additional panic attacks. Agoraphobia is a component of this disorder. 